\title{
Graceful Labeling of Hypertrees
}

\author{
H. El-Zohny ${ }^{1}$, S. Radwan ${ }^{1}$, S.I. Abo El-Fotooh ${ }^{1}$, Z. Mohammed ${ }^{1}$ \\ ${ }^{1}$ Department of Mathematics, Faculty of Science, Al-Azhar University Cairo, Egypt \\ Correspondence: Z. Mohammed, Department of Mathematics, Faculty of Science, Al-Azhar University Cairo, Egypt
}

Received: November 11, 2020 Accepted: December 19, $2020 \quad$ Online Published: January 11, 2021

doi:10.5539/jmr.v13n1p28

URL: https://doi.org/10.5539/jmr.v13n1p28

\begin{abstract}
Graph labeling is considered as one of the most interesting areas in graph theory. A labeling for a simple graph $G$ (numbering or valuation), is an association of non -negative integers to vertices of $G$ (vertex labeling) or to edges of $G$ (edge labeling) or both of them. In this paper we study the graceful labeling for the k- uniform hypertree and define a condition for the corresponding tree to be graceful. A k- uniform hypertree is graceful if the minimum difference of vertices' labels of each edge is distinct and each one is the label of the corresponding edge.
\end{abstract}

Keywords: hypertree, graceful labeling and k, uniform hypertree

\section{Introduction}

Graph labeling is one of the most fascinating areas in graph theory. A graph labeling for a simple graph $G$ (numbering or valuation), is an association of non -negative integers to vertices of $G$ (vertex labeling) or to edges of $G$ (edge labeling) or both of them. The concept of graceful labeling of tree and graph was introduced by Rosa (Rosa, 1967). Golomb (Solomon, 1972), introduced the name "Graceful Labeling" which is also known as "Ringel Kotzig Conjecture" as introduced by Gallian (Gallian, 2000).

The graceful tree conjecture is a famous conjecture in graph theory, which states "for every tree with $m$ edges its vertices can be distinctly labeled using integers $0,1,2, \ldots \ldots, m$ so that the induced edge labels are also distinct".

Rosa, in his original paper proved that the Eulerian graph with number of edges $m \equiv 1(\bmod 4)$ or $\operatorname{m} \equiv 2(\bmod 4)$ cannot be graceful also proved that the cycles $C_{n}$ with $n$ vertices are graceful if and only if $n \equiv 0(\bmod 4)$ or $n \equiv$ $3(\bmod 4)$. The chosen of mod 4 because the graceful labeling of Eulerian graph with $\mathrm{m}$ and $\mathrm{m}+4$ edges appear to be similar, also the cycles of sizes $n$ and $n+4$ appear to be similar in graceful labeling. Aldred and McKay (Aldred \& McKay, 1998) proved that using a computer program that all trees up to 27 vertices are graceful. This result was extended to trees up to 29 vertices by Michael Horton (Michael, 2003).

Consider a connected hypertree $H$ with a vertex set $V(H)$ which is a non-empty set of elements and a set of edges $E(H)$ which its elements are non-empty subsets of $V(H)$. If each edge for a given hypertree $H$ has exactly $k$ vertices with $k \geq 2$ then $\mathrm{H}$ is called $\mathrm{k}$ - uniform hypertree. The order of $H$ is the number of vertices of $V(H)$. In this paper, we proved that the $\mathrm{k}$ - uniform hypertrees are graceful under a certain condition.

\section{Preliminaries}

Definition 2.1 (Rodrigo, 2016): A graceful labeling can be defined of a graph $G$ with $m$ vertices as a vertex integer labeling $f: V \rightarrow[0, m]$ such that $\mathrm{f}$ is injective and the edge labeling for an edge $u v \in E$ is defined by $f(u v)=$ $|f(u)-f(v)|$ is also injective. A graph $\mathrm{G}$ that admits a graceful labeling is called a graceful labeling.

Definition 2.1 (Yalan, 2020): Consider a k-uniform hypertree with at most two vertices whose degrees are not less than 2 in each edge of $H$. Any two edges with at most one common vertex is called a joint vertex, otherwise this vertex is called non- joint.

Definition 2.2: Consider a "k-uniform hypertree"; one can determine an induced tree $T$ from a given hypertree by taking a non-joint vertex in each pendant edge and all joint vertices in order to determine the vertex set $V(T)$ of the corresponding induced tree $T$. Also vertices are connected in $T$ if there are in the same edge of the hypertree.

\section{Main Result}

Consider a "k- uniform hypertree" $H$ with m edges, then $H$ has at most $m(k-1)+1$ vertices if it has exactly one joint vertex between every two edges, also consider its corresponding induced tree $T$ then $H$ is graceful labeled if the minimum difference of vertices labels for a given edge $e \epsilon E(H)$ (with no repetition of edge labels) yields a distinct 
edge labeling of $H$.

That is, if $u, v$ and $w \in V(H)$ and $u, v, w \in e_{i} ; e_{i} \in E(H)$, then the label of this edge $f\left(e_{i}\right)$ is given by:

$$
f\left(e_{i}\right)=\min \left\{\left|f\left(v_{i}\right)-f\left(v_{i+1}\right)\right|, i=1,2,3, \ldots ., k\right\}
$$

Note that, the label for an edge which already exists is excluded when calculating the minimum difference and considering the next minimum one.

Theorem 3.1: The k- uniform hypertree $H, k \geq 2$ and $m \equiv 0(\bmod 4)$ is graceful.

Proof:

We prove the theorem by induction on $k$. At $k=2$, the $\mathrm{k}$ - uniform hypertree is the ordinary graph and similar to induced tree which has the form of caterpillars or paths which are graceful (Cahit, 1975).

Assume that all $\mathrm{k}$ - uniform hypertree with $k=p, p \geq 2$ are graceful. We want prove that all $\mathrm{k}$ - uniform hypertrees with $k=p+1$ are graceful.

Consider a k- uniform hypertree with $k=p$ which vertices and edges satisfies the graceful labeling condition, adding a vertex to each edge such that there exists $m$ distinct vertices to be labeled, then there exists one possible matching to label these vertices and preserves the graceful labeling condition, one possible matching after adding vertex to each edge is to enumerate them consequently through there corresponding edges, and in each case choose the best matching when adding more vertices, this prove the theorem.

\section{Example:}

Consider a 4- uniform hypertree with 4 edges and 13 vertices and its induced tree $\mathrm{T}$, label vertices of each edge so that the minimum difference of vertices labels of each edge is its corresponding label. A graceful labeling of a 4-uniform hypertree and its corresponding incidence tree is shown in fig. 1

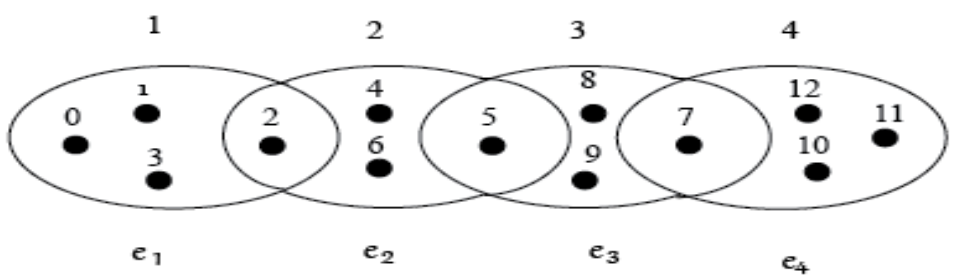

(a)

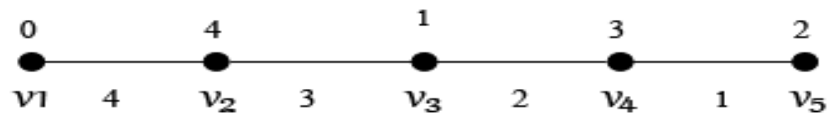

(b)

Figure 1. (a) A graceful labeling of 4- uniform hypertree with 4 edges, (b) A graceful labeling of its incidence tree T

Consider labeling the transformation of a 4- uniform hypertree with 4 edges into 5- uniform hypertree with the same number of edges, we have the graceful labeling shown in fig. 2: 


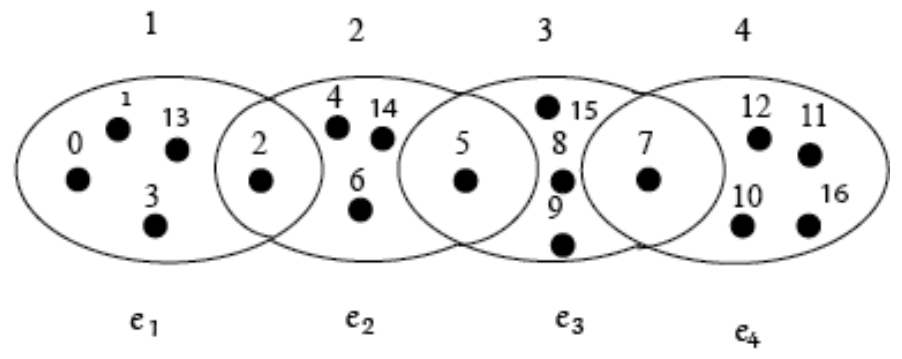

(a)

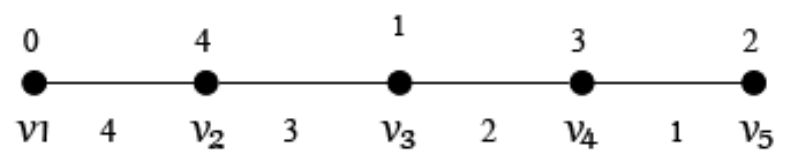

(b)

Figure 2. (a) A graceful labeling of 5- uniform hypertree with 4 edges, (b) A graceful labeling of its incidence tree T

\section{Conclusions}

In this paper, we define a condition for the k- uniform hypertree to be graceful and one can obtain a graceful labeling for the $\mathrm{k}+1$ - uniform hypertree using the graceful labeling of $\mathrm{k}$ - uniform hypertree.

\section{References}

Rosa, A. (1967). On certain valuations of the vertices of a graph. Theory of Graph, 349 - 355.

Solomon, W. G. (1972). How to number a graph. Graph Theory and Computing.

Gallian, J. A. (2000). A dynamic survey of graph labeling Electronic Journal of Combinatorics.

Aldred, \& McKay. (1998). Graceful and harmonious labeling of trees. Bulletin of the Institute of Combinatorics and its Applications, 23, $69-72$.

Michael, H. (2003). Graceful trees: statistics and algorithms. School of computing, University of Tasmania.

Rodrigo, M. Z. (2016). Graceful labeling of graphs. COPPE UFRJ.

Yalan, L., \& Bo, D. (2020). A new method to find the wiener index of hypergraphs. Discrete Dynamics in Nature and Society, 2020. https://doi.org/10.1155/2020/8138942

Cahit, I., \& Cahit, R. (1975). On the graceful numbering of spanning trees. Information Processing Letters, 3(4), 115118. https://doi.org/10.1016/0020-0190(75)90045-9

\section{Copyrights}

Copyright for this article is retained by the author(s), with first publication rights granted to the journal.

This is an open-access article distributed under the terms and conditions of the Creative Commons Attribution license (http://creativecommons.org/licenses/by/4.0/). 